\title{
Comparison of ambulatory blood pressure monitoring and self-blood pressure monitoring for diagnosing white coat hypertension amongst pregnant women
}

\author{
Sandeep Sood ${ }^{1}$ Sirisha Anne ${ }^{1 *}$, Kuldeep Kumar Ashta ${ }^{2}$, Ravi Kumar ${ }^{3}$
}

\author{
${ }^{1}$ Department of Obstetrics and Gynecology, Command Hospital, Lucknow, Uttar Pradesh, India \\ ${ }^{2}$ Department of Internal Medicine, Base Hospital, Delhi Cantt, Delhi, India \\ ${ }^{3}$ Department of Internal Medicine, Armed Forces Medical College, Pune, Maharashtra, India
}

\author{
Received: 01 November 2019 \\ Accepted: 28 November 2019 \\ *Correspondence: \\ Dr. Sirisha Anne, \\ E-mail: siri1407@gmail.com
}

Copyright: () the author(s), publisher and licensee Medip Academy. This is an open-access article distributed under the terms of the Creative Commons Attribution Non-Commercial License, which permits unrestricted non-commercial use, distribution, and reproduction in any medium, provided the original work is properly cited.

\begin{abstract}
Background: White coat hypertension $(\mathrm{WCH})$ is a common and well recognized phenomenon. It is also very prevalent amongst pregnant women and is often diagnosed as chronic/ gestational hypertension leading to unnecessary medications during pregnancy. ABPM is the gold standard for diagnosis of WCH. SBPM is an easy effective and reliable method to measure blood pressure but its efficacy needs to be tested and compared with ABPM in cases of WCH. It is important to compare the two methods in assessing WCH so SBPM can be utilized in cases of $\mathrm{WCH}$, if found useful and efficacious.

Methods: All pregnant women who presented to the ANC were screened for hypertension. Those who were diagnosed to be hypertensive in antenatal clinic and these patients were then admitted for ambulatory blood pressure monitoring (ABPM) for 24 hours and SBPM on 6 hourly bases for 5 days.

Results: The ABPM and SBPB readings were noted, tabulated and compared. It was found that the prevalence of 'WCH' in this study using ABPM and SBPM were 47.368\% (27/54) and 45.614\% (26/54) respectively.

Conclusions: The results in diagnosing WCH using ABPM and SBPM were comparable.
\end{abstract}

Keywords: Ambulatory blood pressure monitoring, Gestational hypertension, Pregnancy, Self-blood pressure monitoring, White coat hypertension

\section{INTRODUCTION}

"White Coat Hypertension" is a well-known entity now days with prevalence as high as $30-35 \% .^{1,2}$ It is equally or probably more common in pregnancy. ${ }^{3,4}$ It is important to identify pregnant women who suffer from this and are wrongly diagnosed as essential hypertension or gestational hypertension. ${ }^{5,6}$ These patients are unnecessarily started on anti-hypertensive medications throughout pregnancy. The anti-hypertension medications when used in pregnancy are also associated with considerable side effects. Ambulatory blood pressure monitoring (ABPM) is the gold standard for diagnosing and assessing uncomplicated hypertension and "White Coat Hypertension". 7-9 ABPM is usually not available in all clinics or at home. Home blood pressure monitoring is the self-measurement of blood pressure by patients. ${ }^{10}$ Self-monitoring/measurement of BP (SMBP) can be done by the patient at home or it can be done in the ward after teaching patient the proper technique of measuring BP. Self/home blood pressure monitoring (SBPM) is a reliable, cheap and easily reproducible alternative method of ABPM in cases of uncomplicated hypertension. ${ }^{11,12}$ Studies have shown that SBPM is superior to blood pressure taken in the clinic in and it is very useful tool in predicting cardiovascular events and mortality. ${ }^{12,13}$ SMBP 
can also be used as an effective way to rule out or exclude $\mathrm{WCH}$, if done in a proper manner. This method is more educative, less expansive, may not require patient admission to ward as it can be done at home and more appropriate for the places where patient loads are high and admitting too many patients to ward is not feasible. ${ }^{13,14}$ The patient efficiency in measuring BP and reliability are the two important factors in implementing this technique. The aneroid sphygmomanometers are widely available now days and are very effective and easy to use; so, it is better to compare ABPM with SBPM as a tool for diagnosis which coat hypertension. There is limited data available where the efficacy of ABPM and SBPM are compared in diagnosing white coat hypertension. It is essential to compare ABPM and SMBP in ruling out or excluding white coat hypertension in pregnant women.

Objective of this study was to do a comparative study to check the efficacy of Ambulatory BP monitoring versus self BP monitoring (SBPM) using an aneroid sphygmomanometer in excluding "white coat hypertension" in pregnant women who were admitted after they were found to have raised BP during their visits to the antenatal clinic (ANC).

\section{METHODS}

This study was a prospective and comparative observational study. This study was done over a period of 1 year 1 month (August 2018 - September 2019) in a Peripheral Hospital with a posted gynecologist and with facilities of ante-natal clinic, family ward and operation theater.

\section{Inclusion criteria}

- All pregnant women with raised BP in ANC after their consent.

\section{Exclusion criteria}

- Those women who didn't gave consent for the study

- Women with age < 18 years and $>35$ years

- Patient already having any other systemic or psychiatric illness

- Patient on any chronic medications (other than the routine medications prescribed in pregnancy) for any other illness.

All pregnant women who attended our ante-natal clinic underwent BP measurement as part of general medical examination using a well calibrated aneroid sphygmomanometer. Those patients who were found to have blood pressure values more than $140 / 90 \mathrm{mmHg}$ were made to rest for $15 \mathrm{~min}$ and $\mathrm{BP}$ was measured again as per the blood pressure protocol. Finally, out of 700 women, 57 were found to have raised blood pressure (> $140 / 90 \mathrm{mmHg}$ ) in antenatal clinic. These patients were then admitted to the family ward for further monitoring and management. Out of these 57 pregnant ladies, 30 were primi-gravida and 27 were multi-gravida. All these patients after admission were trained and were taught how to do record/ measure Self Blood Pressure using an aneroid sphygmomanometer. This training was conducted for two day by the on-duty nursing officer in the ward. Once the nursing officer was convinced that the patients can precisely measure their BP, after that the patients were asked to record their BP 4 times a day at 6 hourly intervals for 5 days as a part of SBPM and simultaneously they were also put on ABPM for $24 \mathrm{hrs}$. ABP is measured using the Holter's machine which measure blood pressure every 20 minutes irrespective of the patient's activity. All routine and specific investigations like complete haemogram, urine tests (for proteinuria), liver and renal function test were done for all patients to assess and rule out the complications of complication of gestational hypertension and preeclampsia. All patients were also screened for fetal growth restriction with ultrasonography (USG). After blood ambulatory blood pressure monitoring for 24 hours and SBPM for 3 days the results of ambulatory blood pressure monitoring were obtained and tabulated in an Excel sheet. The data obtained by using ABPM and SBPM were compared and final results were drawn. A study protocol was made before the commencement of the study delineating the various steps of the study (Figure 1).

\begin{tabular}{|l|}
\hline $\begin{array}{l}\text { Admitted to ward and trained about the prerequisites and how to } \\
\text { record } \mathrm{BP} \text { for } 2 \text { days (till they understood the procedure }\end{array}$ \\
\hline $\begin{array}{l}\text { Patient were put on continuous } \mathrm{BP} \text { measurement using ABPM } \\
\text { for } 24 \text { hours and self } \mathrm{BP} \text { monitoring at } 6 \text { hourly intervals for } 3\end{array}$ \\
\hline $\begin{array}{l}\text { Results of ABPM for } 24 \text { hours and self } \mathrm{BP} \text { monitoring were } \\
\text { compared }\end{array}$ \\
\hline
\end{tabular}

Figure 1: Study protocol.

\section{Statistical analysis}

Assuming the prevalence of $\mathrm{WCH}$ to be around $30-50 \%$ with $95 \%$ confidence interval (CI), alpha (a) error of $5 \%$ and with beta (b) error of $20 \%$. The estimated minimum sample size for the study using the standard formulas was found out to be 48. However, the maximum number of patients who were available during the study duration was included in the study. The data obtained will be filled in MS excel sheets and statistical analysis was done using SPSS Software. 


\section{RESULTS}

Total 57 pregnant women out of 700, who visited to the ante-natal clinic (ANC), were found to have elevated blood pressure. The age groups of these women were as shown in Figure 2.

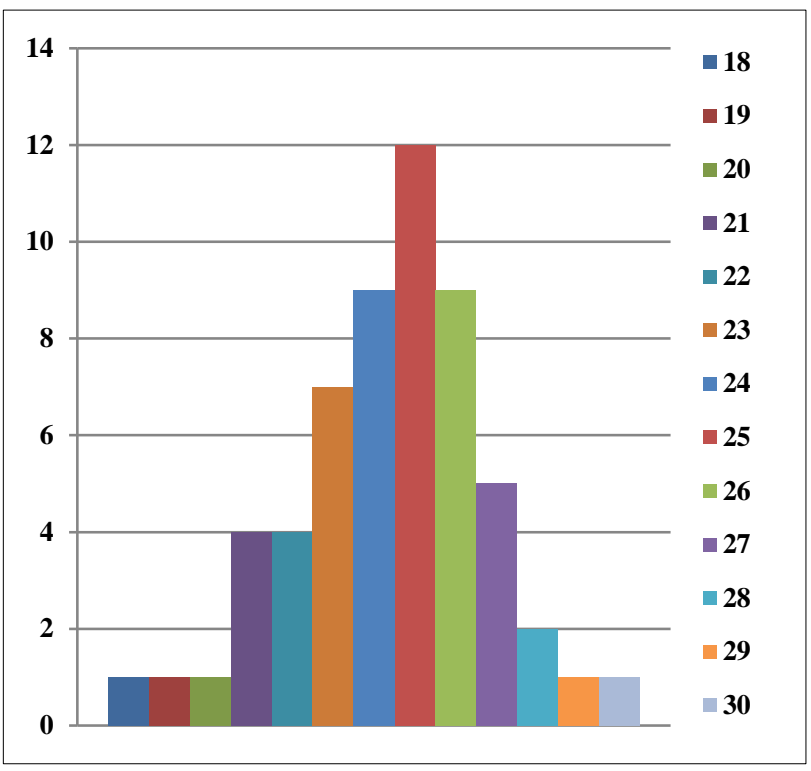

Figure 2: Number of women in various age GP in the study.

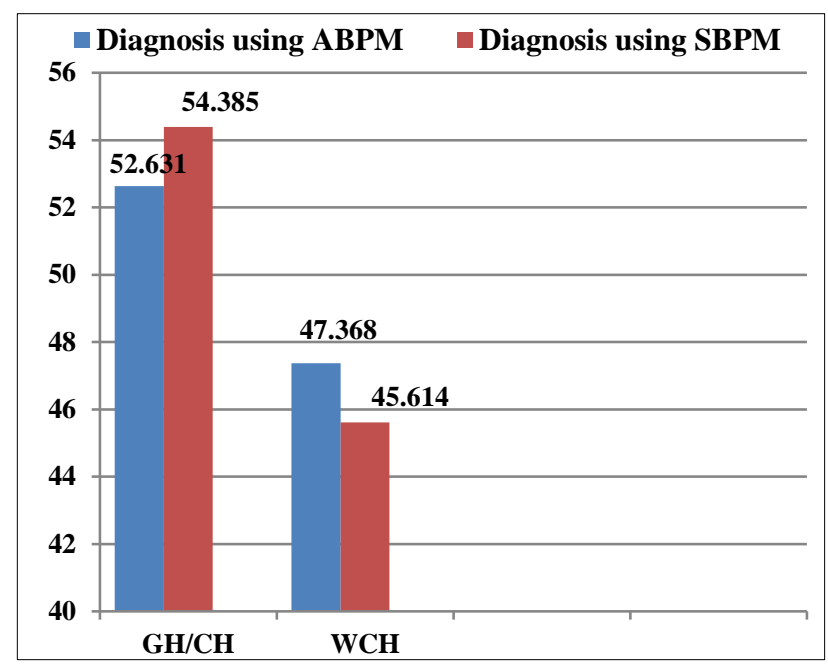

Figure 3: Comparison of results (\%) in diagnosing gestational hypertension (GH)/chronic hypertension (CH) and white coat hypertension (WCH) obtained using ABPM and SBPM.

These women were admitted to family ward for further training on measuring self-monitoring of blood pressure and for ambulatory blood pressure monitoring (ABPM). $52.631 \%(30 / 57)$ patients were diagnosed to have hypertension after ABPM as their average blood pressure was remaining more than $140 / 90 \mathrm{mmHg}$ during the 24 hours of ambulatory blood pressure monitoring on period and $47.368 \%(27 / 57)$ patients were diagnosed as 'white coat hypertension' less than 140/90 mmHg over 24 hours. $54.385 \% \quad(31 / 57)$ patients were diagnosed gestational/chronic hypertension by manual/selfmonitoring of blood pressure of blood pressure in the ward and $45.614 \%(26 / 57)$ patients were diagnosed "White coat hypertension". The comparison of results in diagnosing gestational hypertension (GH)/chronic hypertension $(\mathrm{CH})$ and $\mathrm{WCH}$ obtained using ABPM and SBPM are is shown in Figure 3. Out of the patients who were diagnosed of having hypertension, two patients were detected to have mean blood pressure continuously $>160 / 110 \mathrm{mmHg}$ by ABPM and their BP readings by manual/SMBP were $>150 / 100 \mathrm{mmHg}$. The systolic and diastolic BP tracings of a normo-tensive and a hypertensive pregnant woman obtained by ABPM (using Holter's BP monitoring) are shown in Figure 4 and 5.

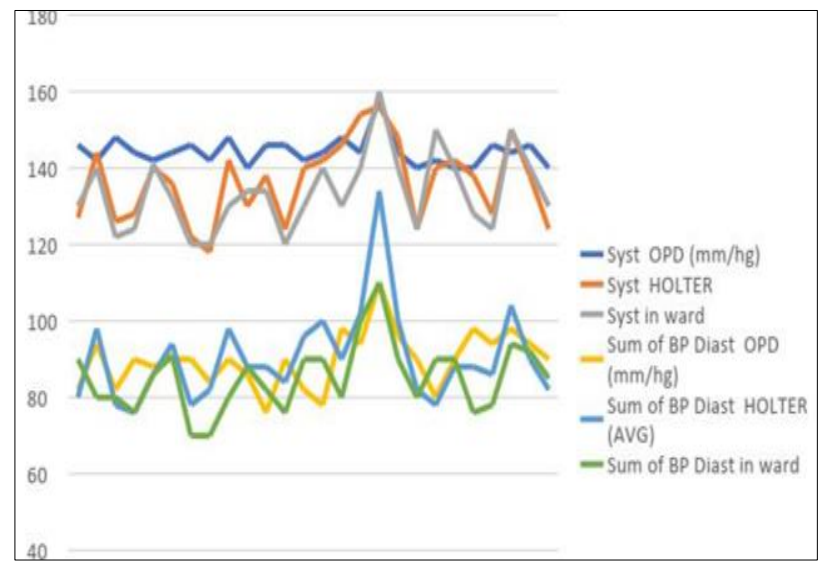

Figure 4: The systolic and diastolic BP tracings of a normo-tensive taken using Holter's BP monitoring.

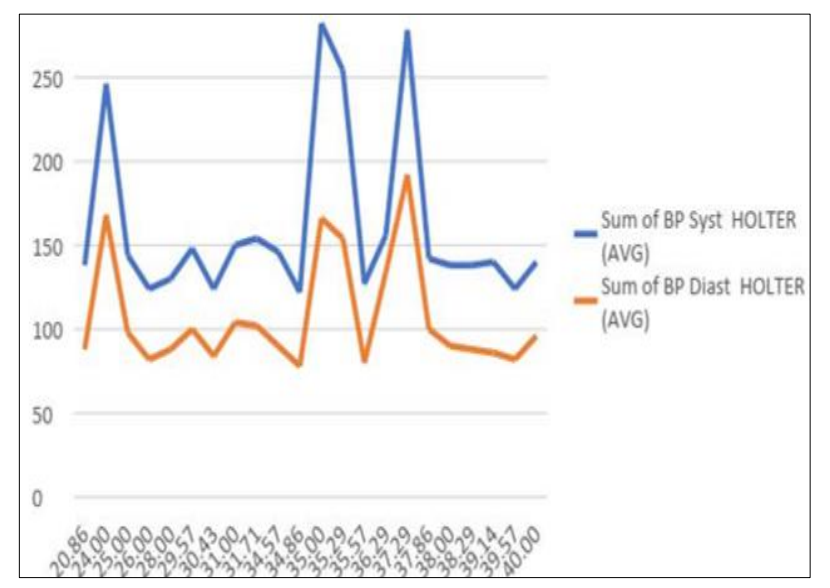

Figure 5: A hypertensive pregnant woman taken using Holter's BP monitoring taken using Holter's BP monitoring.

\section{DISCUSSION}

There are increased risk factors for hypertensive disorders in pregnancy like advanced maternal age, multiple births, diabetes, chronic hypertension, obesity, previous history of preeclampsia, maternal and fetal genetic factors etc 
and these make hypertension the most common medical disorder in pregnancy. ${ }^{14-16}$ This prevalence is further increased by the uncertainty added due to the presence of white coat hypertension ( $\mathrm{WCH})$, which is very common during pregnancy. A recent task force concerning BP measurement and cardiovascular outcomes recommended that "pregnancy is a special indication for ambulatory blood pressure monitoring so white coat hypertension can be effectively ruled out and unnecessarily antihypertensive medications and unnecessary caesarean section can be avoided in pregnancy. ${ }^{17}$ The white coat hypertension is due to reflex activation of the sympathetic nervous system. ABPM is the gold standard for the diagnosis of WCH and uncomplicated hypertension. Studies have suggested that SBPM is a better method for measurement of BP values as compared to the OPD or office blood pressure measurement. ${ }^{18}$

ABPM and SBPM both can give us an almost accurate estimate of BP. Both these methods of BP measurement i.e ABPM and SBPM have strong prognostic significance and theses are effective predictors of the cardio-vascular outcomes related to hypertension. Many studies have been done on ABPM and large amount of data had been generated on it, but not much data is available on SBPM. ABPM and SBPM are complementary tools in evaluation and management of hypertension. ${ }^{19,20}$ ABPM gives us frequent, programmed, and automated BP measurements at regular short intervals over a period of 24 hour irrespective of the patient activity and state. With ABPM the BP changes over short periods can be analyzed. SBPM gives us the repeated measurements of BP over long periods of time as compared to ABPM but it can be tailored as per the convenience and for the shorter durations also. ${ }^{20,21}$ However, repeated performance of ABPM needs special consideration because it is relatively expensive and inconvenient to apply. A preferential and more apt role of SBPM could lies in long term management of hypertension while ABPM is a useful tool for the initial assessment of hypertension, and for the evaluation of relatively high-risk patients.

In WCH and the ABPM and SBPM values are close and co-relatable. $^{21}$ It is even better that in patient with suspected $\mathrm{WCH}$ the $\mathrm{BP}$ is recorded during the day time at home when the patient is much relaxed i.e. by SBPM but the ABPM gives an advantage of providing regular reading at short interval making it a more specific tool for WCH. To measure WCH the SBPM may equal or probably higher sensitivity but lower specificity as compared to ABPM. ${ }^{21}$ There are not many differences in the measured BP values by AMBP and SBPM. The similar results were shown in a study conducted by Dan Hold et al comparing ABPM and SBPM for $\mathrm{WCH}^{21,22}$ There are many studies going on to assess the role of SBPM or HBPM in prognostication and predication of the cardio-vascular mortality due to hypertension whereas ABPM has be proven to be an important toll in predicting the future cardiovascular outcomes due to hypertension. $^{22,23}$ Currently, there is no doubt in considering ABPM as the gold standard or the reference tool for uncomplicated hypertension or $\mathrm{WCH}$ but there is still immense scope to study the SBPM as an effective tool in hypertension. SBPM cannot replace ABPM, but it can be a reliable and cheap alternative to ABPM in many aspects related to hypertension. Most of the studies done to compare the ABPM and SBPM have concluded that the finding of ABPM and SBPM are co-relatable and the ABPM values are reproducible on SBPM without much differences and they have also and they have also given consensus on SBPM being not a replacement but a complementary or alternative tool for diagnosis and evaluation of hypertension and related complications. ${ }^{24.25}$

Limitations of the study were the sample size of the study has been small, owing to the fact that the study was done at a peripheral hospital. Further larger trials need to be conducted to reinforce these facts.

\section{CONCLUSION}

Detecting gestational hypertension accurately is very important for good antenatal care. 'White coat hypertension' is also a very well-known entity in this clientele as patients are extremely anxious about their well-being. $48.15 \%$ patients in the study who were initially diagnosed as gestational hypertension were later found to have 'white coat hypertension' by ABPM. Hence, we recommend all patients found to be having raised $\mathrm{BP}$ recording in OPD should be further evaluated using ABPM which can used in ward or at their home and then interpret the results. It would avoid unnecessary admission and medication to the patients.

\section{Funding: No funding sources \\ Conflict of interest: None declared}

Ethical approval: The study was approved by the Institutional Ethics Committee

\section{REFERENCES}

1. Brown MA, Davis GK. Hypertension in pregnancy. In: Mancia G, Chalmers J, Julius S, et al, editors. Manual of Hypertension. London: Churchill Livingstone; 2002:579-598.

2. Brown MA, Mangos G, Greg D, Homer C. The natural history of white coat hypertension during pregnancy. Int J Obstet Gynecol. 2005;112:601-6.

3. Franklin SS, Thijs L, Hansen TW, O'Brien E, Staessen JA. White-Coat Hypertension: New Insights from Recent Studies. Am Heart Assoc J. 2013;62(6):982-6.

4. Higgins JR, de Swiet. M. Blood -pressure measurement and classification in pregnancy. Lancet. 2001;357:131-5.

5. Brown MA, Lindheimer MD, de Swiet M, Van Assche A, Moutquin JM. The classification and diagnosis of the hypertensive disorders of pregnancy: Statement from International Society for the Study of 
Hypertension in Pregnancy (ISSHP). Hyper Preg. 2001;20:x-xiv.

6. Contard S, Chanudet X, Coisne D, Battistella P, Marichal JF, Pitiot M, Gaudemaris R, Ribstein J. Ambulatory monitoring of blood pressure in normal pregnancy. Am J Hypertens. 1993:880-4.

7. Halligan A, O’Brien E, O’Malley K, Mee F, Atkins N, Conroy R, Walshe JJ, Darling M. Twenty-four hour ambulatory blood pressure measurement in a primigravid population. J Hypertens. 1993;11:869-73.

8. Hermida RC, Ayala DE, Mojón A, Fernández JR. Time-qualified reference values for ambulatory blood pressure monitoring in pregnancy. Hypertens. 2001;38:746-52.

9. Stergiou GS, Asayama K, Thijs L, Kollias A, Niiranen TJ, Hozawa A, et al. Prognosis of WhiteCoat and Masked Hypertension. Hypertens. 2014;63:675-82.

10. Owens P, Atkins N, O'Brien E. Diagnosis of white coat hypertension by ambulatory blood pressure monitoring. Hypertens. 1999;34(2):267-72.

11. Reynolds K, Bowling CB, Sim JJ, Sridharan L, Harrison TN, Shimbo D. The utility of ambulatory blood pressure monitoring for diagnosing white coat hypertension in older adults. Current Hypertens Reports. 2015;17(11):86.

12. Boggia J, Hansen TW, Asayama K, Luzardo L, Li Yan, Staessen JA. White-coat hypertension on automated blood pressure measurement: implications for clinical practice. Eu J Cardiovasc Med. 2011;1(4):17-21.

13. Mc Grath BP. Home monitoring of blood pressure. Aust Prescr. 2015;38:16-9.

14. Hernández-Díaz S, Toh S, Cnattingius S. Risk of pre-ecclampsia in first and subsequent pregnancies: prospective cohort study. BMJ. 2009;338:b2255.

15. Duckitt K, Harrington D. Risk factors for preeclampsia at antenatal booking: systematic review of controlled studies. BMJ. 2005;330:565.

16. Cnattingius S, Reilly M, Pawitan Y, Lichtenstein P. Maternal and fetal genetic factors account for most of familial aggregation of preeclampsia: a population-based Swedish cohort study. Am J Med Genet. 2004;130A:365-71.
17. Bar J, Maymon R, Padoa A, Wittenberg C, Boner G, Ben-Rafael Z, et al. White coat hypertension and pregnancy outcome. J Human Hypertens. 1999;13(8):541.

18. Zhang L, Li Y, Wei FF, Thijs L, Kang YY, Wang S, et al. Strategies for classifying patients based on office, home, and ambulatory blood pressure measurement. Hypertens. 2015;65(6):1258-65.

19. Ghuman N, Campbell P, White WB. Role of ambulatory and home blood pressure recording in clinical practice. Curr Cardiol Rep. 2009;11(6):414-21.

20. Sheppard JP, Fletcher B, Gill P, Martin U, Roberts N, McManus RJ. Predictors of the home-clinic blood pressure difference: a systematic review and metaanalysis. Am J Hypertens. 2015;29(5):614-25.

21. Celis H, Den Hond E, Staessen JA. Selfmeasurement of blood pressure at home in the management of hypertension. Clin Med Res. 2005;3(1):19-26.

22. Den Hond E, Celis H, Fagard R, Keary L, Leeman M, O'Brien E, et al. Self-measured versus ambulatory blood pressure in the diagnosis of hypertension. J Hypertens. 2003;21(4):717-22.

23. Kim SG. Clinical implications of ambulatory and home blood pressure monitoring. Korean Circ J. 2010;40:423-31.

24. Bellomo G, Narducci PL, Rondoni F, Pastorelli G, Stangoni G, Angeli G, et al. Prognostic value of 24hour blood pressure in pregnancy. JAMA. 1999;282:1447-52.

25. Moutquin JM, Rainville C, Giroux L, Raynauld P, Amyot G, Bilodeau R, et al. A prospective study of blood pressure in pregnancy: prediction of preeclampsia. Am J Obstet Gynecol. 1985;151(2):191-6.

Cite this article as: Sood S, Anne S, Ashta KK, Kumar R. Comparison of ambulatory blood pressure monitoring and self-blood pressure monitoring for diagnosing white coat hypertension amongst pregnant women. Int J Reprod Contracept Obstet Gynecol 2020;9:274-8. 